W2F-(5)-3

\title{
Investigation of Sum-Frequency Generation of Chiral Molecules in Solutions
}

\author{
M. Hayashi ${ }^{\text {a) }}$, S. H. Lin ${ }^{\text {b) }}$, and Y. R. Shen ${ }^{\text {c) }}$ \\ a) Center for Condensed Matter Sciences, National Taiwan University, \\ Taipei 106 Taiwan, ROC \\ b) Institute of Atomic and Molecular Sciences, Academia Sinica, \\ P. O. Box 23-166, Taipei 106 ROC \\ c) Department of Physics, University of California and Material Sciences Division, \\ Lawrence Berkeley National Laboratory, Berkeley, California 94720 \\ *: atmyh@ccms.ntu.edu.tw
}

\begin{abstract}
A main purpose of this paper is to examine the origin and mechanism of non-linear optical activity in solution. For this purpose, within the dipole-dipole formalism of SFG, we study the non-Condon effect and the effect of the breakdown of the Born-Oppenheimer approximation. We find that in order to determine which mechanism is responsible for this SFG it is necessary to compare the SFG band-shape functions on surfaces and in solutions.

Quite recently, visible-visible Sum Frequency Generation (SFG) signals from 1,1'-Bi-2-naphthol (BN) in a bulk BN solution have been observed. [1,2] To understand the origin of the SFG activity of BN in solution, we shall theoretically examine a SFG mechanism of chiral molecules in a bulk solution. For this purpose, we shall employ the Herzberg-Teller theory to study the non-Condon effect; we shall also study the effect of the breakdown of the Born-Oppenheimer adiabatic approximation on this nonlinear optical activity in solution. The SFG beyond the dipole-dipole approximation like the dipole-magnetic dipole approximation and the magnetic dipole-magnetic dipole approximation will also be explored in this paper.
\end{abstract}

[1] M. A. Belkin, S. H. Hau, X. Wei, and Y. R. Shen, Phys. Rev. Lett. 87, art. no. 113001 (2001).

[2] P. Fischer, K. Beckwitt, F. W. Wise, A. C. Albrecht Chem. Phys. Lett. 352, 463 (2002). 